Revue d'histoire de l'Amérique française

RAS REVUE D.HISTOIRE DE L'AMÉRIQUE FRANÇAISE

\title{
Les débuts de l'enseignement de l'histoire et de la géographie au Petit Séminaire de Québec (1765-1880) (suite et fin)
}

\section{Pierre Savard}

Volume 16, numéro 2, septembre 1962

URI : https://id.erudit.org/iderudit/302193ar

DOI : https://doi.org/10.7202/302193ar

Aller au sommaire du numéro

Éditeur(s)

Institut d'histoire de l'Amérique française

ISSN

0035-2357 (imprimé)

1492-1383 (numérique)

Découvrir la revue

Citer cet article

Savard, P. (1962). Les débuts de l'enseignement de l'histoire et de la géographie au Petit Séminaire de Québec (1765-1880) (suite et fin). Revue d'histoire de l'Amérique française, 16(2), 188-212. https://doi.org/10.7202/302193ar d'utilisation que vous pouvez consulter en ligne. 


\section{LES DÉBUTS DE L'ENSEIGNEMENT DE L'HISTOIRE ET DE LA GÉOGRAPHIE AU PETIT SÉMINAIRE DE QUÉBEC * (1765-1880) \\ (suite et fin)}

CHAPITRE TROISIÈME

\section{LE TRIOMPHE DE L'HISTOIRE DANS L'ENSEIGNEMENT} (1845-1870)

On assiste en Occident au milieu du $\mathrm{XIX}^{\mathrm{e}}$ siècle à ce qu'on a appelé justement une "inflation des valeurs historiques ». ${ }^{1}$ Il devient de mode d'emprunter alors à l'Histoire des précédents, des exemples et des arguments. Au fond tout cela correspond à un puissant mouvement de pensée qui cherche dans l'Histoire et l'idée d'évolution, l'explication suprême. Depuis Hegel, Lamarck, Darwin et Marx tous les problèmes se posent en termes historiques. Et le couronnement des sciences humaines apparait dans la philosophie de l'histoire. Un des plus brillants et des plus caractéristiques des penseurs de l'époque écrira alors:

Voir, pour début de cet article, notre Revue, XV: 509-525; XVI: 43-62.

* L'auteur a terminé la rédaction de ce mémoire en décembre 1959. Depuis, ont paru trois articles importants en rapport avec ce sujet: M. Antonio Drolet a publié dans la Revue d'Histoire de l'Amérique française de mars 1961 un article intéressant sur la bibliothèque des Jésuites, bibliothèque dont plusieurs ouvrages se retrouvent au Petit Séminaire. M. Louis-Edmond Hamelin, d'autre part, a publié dans les Cahiers de Géographie, $4 \mathrm{e}$ année, $\mathrm{N}^{\circ} 8$ (avril-septembre 1960) la première partie d'une Bibliographie annotée concernant la pénétration de la géographie dans le Québec. La seconde partie du travail de M. Hamelin intitulée Notes et Documents a été polycopiée dans la collection des T.I.G.U.L. (Travaux de l'Institut de Géographie de l'Université Laval) N 8, Québec, 1959-1960.

L'auteur tient à exprimer ici sa gratitude à M. Claude Galarneau, professeur agrégé à la faculté des Lettres de Laval, qui a dirigé ce travail. M. Louis-Edmond Hamelin lui a aimablement communiqué une partie encore manuscrite de l'étude citée supra, tandis que Mgr Arthur Maheux lui a fourni de précieux renseignements sur les archives du Séminaire de Québec et leurs problèmes.

1 Henri-Irénée Marrou, De la connaissance historique, 13. 
La doctrine qui aura suffisamment expliqué l'ensemble du passé obtiendra inévitablement par suite de cette seule épreuve, la présidence mentale de l'avenir. ${ }^{2}$

Ce mouvement semble avoir influencé tous les penseurs du temps, à quelque enseigne qu'ils logeassent. Mais on étudie surtout l'histoire nationale car la question des nationalités reste à l'ordre du jour. On ne saurait exagérer le rôle des études et de la conscience historiques dans le mouvement des nationalités. Le $\mathrm{XIX}^{e}$ siècle mérite d'être appelé non seulement le siècle de l'histoire mais celui de l'histoire nationale. ${ }^{3}$ Les programmes de cette époque sont bien entendu envahis par l'histoire. Ainsi, dans les nouveaux programmes de 1852 en France, l'histoire nationale occupe la première place. ${ }^{4}$ Monseigneur Dupanloup pourra écrire quelques années plus tard:

L'étude et l'enseignement de l'Histoire ont pris depuis quelques années parmi nous une importance et un développement démesuré ... une étude négligée et presque oubliée est devenue bientôt une étude dominante et presque exclusive ... et aujourd'hui il n'est pas une faculté, pas un collège, par un examen où cet enseignement ne se trouve en première ligne. ${ }^{5}$

A cet engouement de la vieille Europe pour l'histoire correspond, au Canada, l'enthousiasme suscité par les travaux de Garneau dont l'Histoire $d u$ Canada, publiée en 3 volumes de 1845 à 1852, marque le triomphe définitif chez nous du genre historique. Et l'histoire envahit alors la littérature. La poésie lui emprunte ses sujets, le roman historique commence à pulluler, le drame historique sera bientôt à la mode. Il se produit aussi depuis Garneau un glissement dans la mentalité canadienne-française. Comme on l'a fait remarquer, le dogme de la supériorité nationale apparaît chez le Canadien français, jusque-là souvent

\footnotetext{
2 Auguste Comte, cité par Marrou, op. cit., 13.

3 Marcel Reinhard, Histoire des Histoires de France dans Histoire de France..., I: 21. II : 79.

4 Octave Gréard, Education et instruction. Enseignement secondaire ..., II : 15.

5 Monseigneur l'évêque d'Orléans, De la haute éducation intellectuelle,
} 
réduit à la défensive. ${ }^{6}$ Bientôt, un autre historien de grande valeur, l'abbé Ferland, viendra compléter et corriger certaines vues de Garneau en particulier au sujet de l'histoire religieuse. Mais après ces deux grands noms, "l'histoire piétine... ce ne sont que narrations sans relief, travaux de vulgarisation, biographies d'amateurs ou monographies régionales » à la méthode souvent insuffisante. ${ }^{7}$ Quoique l'histoire reste en voyage, il faudra attendre longtemps, c'est-à-dire jusqu'à Chapais et l'abbé Groulx, pour voir apparaître d'autres synthèses de valeur.

Un coup d'œil sur les programmes d'étude de l'époque nous révèle la place considérable qu'y occupe l'histoire. Lors du premier concours du Baccalauréat en 1853, une des six épreuves écrites consiste en une composition sur un sujet d'histoire ou de géographie. L'examen dure quatre heures et le résultat compte pour 24 points soit un cinquième du total. La liste des sujets alors proposés peut donner une idée des problèmes historiques et géographiques auxquels on s'efforce d'intéresser les jeunes du temps:

\section{Comment Jules César parvint-il à la souveraine puissance ? \\ Raconter les principaux événements qui se sont écoulés depuis la convocation des Etats Généraux jusqu'à la mort de Louis XVI. \\ Nommer les principales villes d'Angleterre et dire quelque chose sur chacune d'elles. \\ Raconter la découverte du Canada. ${ }^{8}$}

On étudie également à l'époque la géographie historique appelée géographie ancienne ${ }^{9}$ et la méthodologie de l'histoire à l'occasion des préceptes littéraires. ${ }^{10}$ Enfin nombre d'historiens figurent au programme des auteurs français, tels Voltaire (Le siècle de Louis XIV, Histoire de Pierre le Grand, Histoire de Charles VII), Montesquieu (Grandeur et décadence des Ro-

${ }^{6} \mathrm{Mgr}$ Emile Chartier, Au Canada-Français. La vie de l'esprit, 1760$1925,78$.

7 Auguste Viatte, Histoire littéraire de l'Amérique française, 108.

8 L'Abeille, 3 novembre 1853.

9 Programmes du Baccalauréat ès-arts à l'Université Laval, Programme de Géographie 5.

10 Ibid., Programme de littérature, 4. 
mains), Fénelon (Lettre à l'Académie), Bossuet (Discours sur l'histoire universelle, Histoire des Variations). Bien entendu les historiens anciens sont toujours à l'honneur tels Salluste, César, Tite-Live, Xénophon, Thucydide, etc....11

$\mathrm{Au}$ Petit Séminaire, l'esprit historique s'infiltre partout. C'est à cette époque qu'apparaît l'Abeille, journal imprimé par les élèves eux-mêmes. La place de l'histoire y est considérable. Sans doute faut-il y voir l'influence discrète d'un des fondateurs et premier éditeur, Charles-Honoré Laverdière, ${ }^{12}$ mais cela semble répondre aussi au goût de l'époque. Même à l'occasion de questions d'actualité le journal a l'habitude d'envisager les problèmes dans une perspective historique. C'est ainsi que lors de la guerre de Sécession, on en profite pour servir aux lecteurs une esquisse de l'histoire de la république voisine. ${ }^{13}$ L'expédition du Mexique fournit également l'occasion d'une « analyse sur l'histoire de ce pays pendant la domination Espagnole dans cette contrée et les cinquante années de troubles et d'anarchie qui ont suivi ». ${ }^{14}$ En 1848, au moment où «presque partout des constitutions sont discutées, demandées ou exigées, promises ou refusées, modifiées ou renversées », un auteur soumet une « Esquisse [historique] de la constitution du royaume d'Angleterre $\gg^{15}$

Les sociétés ou cercles qui fleurissent à l'époque consacrent beaucoup de leurs énergies à l'histoire. La société Laval, cercle d'éloquence fondé en 1851, place la composition historique parmi les cinq types de travaux proposés à ses membres. En fait l'histoire semble le genre de prédilection, du moins au début. Tous les sujets possibles y sont exposés et discutés depuis la dispersion des Acadiens jusqu'au problème traditionnel: «Quel fut le plus

11 Ibid., Programme d'histoire de la littérature, 3s.

12 Le rôle exact de Laverdière dans la fondation et l'animation de l'Abeille reste à étudier. Dans le premier numéro (27 juillet 1848) il nous parle d'un article-prospectus \& renfermant notre plan, nos principes par rapport aux matières que nous croyons avoir à traiter $\gg$. Cet article jamais publié aurait pu éclairer le problème.

13 L'Abeille, X (1861-62), nos 13 à 21, soit du 27 mars au 22 mai 1862.

14 Ibid., 6, 13, 20 et 27 février 1862. 1849.

15 Ibid., $X(1848-49)$, nos $6,7,8$ et 17 , soit de novembre 1848 à janvier 
grand des deux monarques: Charles-Quint ou François 1er ? ${ }^{16}$ En 1852, on assiste à la fondation d'une société qui se donne pour but spécifique «de répandre parmi nous la lecture de l'histoire et de nous affermir dans cette intéressante partie de nos études ».17 Le bilan des travaux à la fin de la première année d'activité est impressionnant. Les 15 membres ont tous lu Rollin, ils ont « produit l'analyse de 150 traits d'histoire romaine qui se lit au réfectoire outre 40 discours, dont environ 10 ayant trait à l'histoire moderne ».18 Il s'agit de la Société Maizerets qui groupe des élèves de la petite Salle. Des sociétés de ce genre semblent avoir connu beaucoup de vogue dans les autres collèges classiques de l'époque. En 1842, Antoine Gérin-Lajoie, étudiant en philosophie à Nicolet, fonde la société littéraire du Séminaire de Nicolet. ${ }^{19}$ Le préfet des études du Collège de l'Assomption pour sa part fondera en 1853 l'Académie française, cercle littéraire ouvert aux élèves des classes supérieures. ${ }^{20}$

A toutes ces activités reliées à l'histoire viennent s'ajouter les lectures au réfectoire, la plupart du temps tirées d'ouvrages historiques ${ }^{21}$ et les longues promenades, les jours de congé, à travers le vieux Québec. ${ }^{22}$ De plus les élèves des classes supérieures ont le privilège d'assister aux conférences publiques qui se donnent presque tous les hivers à l'Université. On sait que c'est à l'occasion de ces cours publics, de 1853 à 1862, que l'abbé Ferland livrera les résultats de ses travaux sur le régime français. Soulignons également la proximité pour les élèves du Séminaire des musées d'archéologie, de peinture et de numismatique inaugurés à Laval durant cette période. A vrai dire les élèves

16 On trouvera un historique utile de la société Laval dans l'Abeille, XIII (1879-80), nos $16,19,20,25,28,29$ et 33 , soit du 31 décembre 1879 au 29 avril 1880 .

17 Ibid., 1 juillet 1852.

18 Loc. cit.

19 [Anonyme], Le Séminaire de Nicolet, 43.

20 Abbé Anastase Forget, 1833 - Un siècle - 1933. Histoire du Collège de l'Assomption, 172.

21 J. E. Roy, Souvenirs d'une Classe au Séminaire de Québec (1867$1877), 272 \mathrm{~s}$.

22 Ibid., 409. Voir un compte rendu d'une promenade historique de rhétoriciens avec leur professeur d'histoire du Canada, dans l'Abeille, 19 novembre 1879. Voir aussi: O. Pelletier, Mémoires, 55. 
intéressés à l'histoire étaient comblés. Et il suffit de lire les souvenirs de Joseph-Edmond Roy, écolier à cette époque, pour constater que certains de ces derniers savaient tirer parti au maximum de ces possibilités. Un goût frénétique de l'histoire avait envahi le vieux Séminaire. Ce goût, on s'en doute, était stimulé par des professeurs, véritables savants, au premier rang desquels il faut placer l'abbé Laverdière. ${ }^{23}$ Figure bien attachante que celle de cet homme si diversement doué. Dessinateur brillant, mathématicien, musicien et surtout archéologue et historien, son seul malheur fut d'écrire avec difficulté à une époque où le style était trop souvent le critère suprême de jugement d'un intellectuel. Aussi sa contribution importante à l'histoire du Canada fut-elle souvent méconnue. Rappelons seulement qu'il fut un des principaux artisans de la réédition des Relations des Jésuites. Ce travailleur acharné, mort relativement jeune, édita aussi les Oeuvres de Champlain. Travail impeccable et méticuleux qui a fait dire à l'historien George Wrong:

Too much cannot be said in praise of the high character of Laverdiere's work. He gave a good part of his life to the task and did it with the meticulous accuracy and desire for perfection that the poet Browning describes in the scholar of his Grammarian's Funeral. I never think of Laverdiere and his work without associating him with that creation. $\mathrm{He}$ died, alas, when still comparatively young. Rarely shall we see his like. ${ }^{24}$

Un ami de l'abbé Laverdière, l'abbé L.-O. Gauthier, continuateur de la Géographie de l'abbé Holmes, se révélera aussi à l'époque un professeur de valeur et laissera un excellent souvenir chez ses anciens élèves. Savant à la curiosité insatiable, il pos-

23 Sur la carrière et l'œuvre de l'abbé Laverdière (1826-1873) il n'y a pas de travaux d'envergure. On peut consulter toutefois parmi plusieurs articles et brochures, l'éloge public prononcé en 1878 par Hubert LaRue et reproduit partiellement dans ses Mélanges historiques et littéraires [...], II: 145-151, la brochure de Faucher de Saint-Maurice tirée de l'Opinion publique du 27 mars 1873 et l'abbé Auguste Gosselin, Le vrai monument de Champlain: ses cuvres éditées par Laverdière dans MSRC (3ième série), II (1908) : 3-23. Le journal de Laverdière et sa correspondance sont conservés aux Archives du Séminaire de Québec.

${ }^{24}$ Cité dans le Centenaire de l'histoire du Canada de François-Xavier Garneau [...], 225. 
sédait l'hébreu et s'était mis à l'étude des hiéroglyphes égyptiens. «On aurait dit que les découvertes de Champollion l'empêchaient de dormir », a-t-on pu écrire. ${ }^{25}$ Il publia un abrégé d'histoire du Canada estimé. ${ }^{26}$ L'abbé Gauthier fut le premier professeur d'histoire du Canada en titre de façon régulière à partir de 1865. Avant lui les abbés Holmes, Taschereau et Laverdière avaient eu le titre de professeur d'histoire, mais de façon occasionnelle. Quant à l'histoire universelle, elle aura son professeur spécial pour de bon en 1871. Notons qu'à ce moment seuls l'anglais, le dessin et la musique comptaient des professeurs spéciaux respectivement depuis 1814, 1833 et $1834 .{ }^{27}$

L'abondante documentation qui nous est restée permet de connaître la conception de l'histoire d'alors, les auteurs qu'on estimait et lisait, voire les méthodes d'enseignement. Plusieurs articles de l'Abeille nous renseignent particulièrement sur l'idée qu'on se fait à l'époque de la nature et du rôle de l'histoire. Un éditorial non signé en 1849 déclare que l'histoire est un «oracle du présent » sans lequel nous ne pourrions «suivre dans tous ses ravages le flot impétueux qui vient de s'abattre sur l'Europe ». Oracle aussi de l'antiquité qu'elle nous dévoile et de l'avenir qu'elle nous découvre, l'histoire assure l'avantage de servir d'excellentes leçons de morale. ${ }^{28}$ Un correspondant de l'Assomption célèbre en ces termes l'étude de cette discipline:

C'est là que le penseur peut donner libre cours à ses réflexions, c'est là qu'il verra la providence et la justice de Dieu écrites non sur des monuments mais sur le monde entier. ${ }^{29}$

Enfin, on écrit à cette époque que l'étude de l'histoire, «après celle de la religion est la plus propre à orner l'esprit et à former le cœur $\gg^{30}$ La grande histoire, celle qui plane volon-

25 L'Abeille, 14 octobre 1880.

26 Sur l'abbé Gauthier (1840-1880) lire la notice nécrologique parue dans l'Abeille du 14 octobre 1880 .

27 ASQ, Supérieurs, Directeurs, officiers et professeurs du Séminaire de Québec de 1833 à 1860 .

28 L'Abeille, 8 février 1849.

29 Ibid., 1 juillet 1862.

30 Ibid., 16 juin 1853. 
tiers très haut au-dessus des événements, fleurit. Tel ce discours sur l'histoire moderne, prototype du genre, composé par le supérieur du Collège de Saint-Hyacinthe et reproduit dans l'Abeille de $1849 . .^{31}$ Le morceau qui ne manque pas d'éloquence nous propose une "revue rapide des faits saillants de l'histoire moderne en examinant quelle a pu être la raison de leur accomplissement sous le point de vue providentiel ». La perspective providentialiste gagne également l'étude de l'histoire nationale sur laquelle nous reviendrons plus loin. Un article de l'Abeille en 1848 dit explicitement:

Cette Providence adorable qui a toujours veillé sur notre pays pour le protéger n'a-t-elle pas aussi quelque attrait pour le lecteur? Le Canada est-il affligé par toutes les horreurs de la famine? Aussitôt Dieu lui envoie une armée qui s'empare du pays et fait renaître l'abondance. Une révolution infernale se prépare-t-elle en France ? De nouveau le Canada est arraché de ses mains et transmis à l'Angleterre qui le protège encore aujourd'hui de sa puissante égide. ${ }^{32}$

On attend beaucoup de l'histoire, école de morale. Un article de l'Abeille en 1853 souligne cette fonction de la connaissance historique en ces termes:

Toutes les actions des hommes, quelque différentes qu'elles soient, ne sont pourtant composées que d'un petit nombre de circonstances et de motifs, quand une fois l'âme a formé son jugement sur ces circonstances et ces motifs, il lui est bien aisé de transporter les règles qu'elle s'est faites en lisant l'histoire, de les appliquer aux occasions et aux affaires qui arrivent tous les jours..$^{33}$

Le même auteur s'en était pris dans un article précédent à l'histoire-érudition qui, au lieu de faire apprendre aux étudiants

la véritable morale, par des réflexions qu'on leur devrait faire faire sur les endroits les plus singuliers

31 Ibid., 27 novembre et 20 décembre 1849. Le discours prononcé en 1841 est aussi reproduit dans James Huston, Le Répertoire national [...], II : 208-219.

32 Ibid., 1 mars 1849.

33 Ibid., 1 mars 1853. 
et les plus instructifs, [..] ne leur sert qu'à se faire accroire à eux-mêmes, et aux ignorants comme eux, qu'ils savent quelque chose, pendant qu'ils ne savent rien. ${ }^{34}$

Nous avons la bonne fortune de posséder quelques témoignages sur les méthodes d'étude et d'enseignement de l'époque. Ainsi, sous la forme d'une lettre à un confrère, un élève du temps donne des conseils sur l'étude de l'histoire. D'abord il faut lire les grands ouvrages, telle l'Histoire ancienne de Rollin. Et lorsqu'on lit un livre, on doit prendre soin de commencer par la fin, c'est-à-dire par la table des matières. Puis, vient la préface, «parce que, comme on nous a dit, l'auteur y explique le plan de son livre et le but qu'il s'est proposé ...»35 Dans un autre article, le même collaborateur explique comment on fabrique des tableaux chronologiques et comment on analyse une lecture. ${ }^{36}$

Le programme fait encore une large part à l'histoire ancienne qui s'étend alors jusqu'à 476 tandis que l'histoire moderne, comme nous l'avons vu, a remplacé l'étude de l'histoire de France et celle d'Angleterre. Quant au Moyen Âge, il est l'objet du plus grand mépris comme en témoigne cet extrait d'un article publié en 1853 qui, en parlant de 476, nous dit:

C'est là que commence l'époque de la barbarie. L'invasion jette un voile épais sur tout ce qui est science et beaux-arts; et elle plonge la société humaine dans une nuit profonde, qui ne se dissipe que 1000 ans plus tard, devant la renaissance des lettres. ${ }^{37}$

Il peut être intéressant de se demander quels étaient les auteurs en vogue parmi les historiens de ce temps. Un bref examen du contenu de la bibliothèque du Séminaire nous apprend que ce n'est qu'après 1840 que sont connus les grands historiens français: Barante (surtout lu comme critique littéraire), Guizot et Thiers. Michelet pour sa part est à peu près ignoré tandis que les œuvres de Duruy foisonnent sur les rayons.

34 Ibid., 18 janvier 1853.

35 Ibid., 15 mars 1853.

36 Ibid., 5 avril 1853.

37 Ibid., 16 juin 1853. 
Taine ne sera connu, semble-t-il, qu'après 1890 et encore, sous forme de morceaux choisis. Mais ce sont avant tout les écrivains catholiques qui recueillent les suffrages, en particulier Ozanam et Montalembert. La bibliothèque du Séminaire possède la plupart des éditions de ces deux derniers auteurs. C'est ainsi que nous avons retrouvé 6 éditions de l'Histoire de Sainte Elisabeth de Hongrie parues et probablement acquises durant la période que nous étudions ici.

Quelques articles de l'Abeille nous apportent également des jugements sur les historiens de l'époque. A l'occasion de la mort de Macaulay, on reproduit en trois livraisons un long et élogieux article tiré de l'Ami de la Religion. ${ }^{38}$ Ailleurs, un compte rendu de récentes acquisitions de la nouvelle Bibliothèque de l'Université dû probablement à la plume de l'abbé Laverdière, alors bibliothécaire, critique quelques œuvres. L'Histoire $d u$ Consulat et de l'Empire de Thiers est qualifiée de «grand ouvrage» quoique l'auteur «sacrifie encore quelque fois à certains préjugés de son siècle ». On signale les œuvres « si catholiques» de Foisset et d'Ozanam, "l'Histoire de la révolution d'Angleterre par Guizot, le Directoire et les Ducs de Bourgogne par Barante». On réserve une mention on ne peut plus élogieuse à l'Histoire de Louis XVII par De Beauchesne:

Chef d'œuvre de patience, de sensibilité et de jugement... déclare l'auteur de l'article qui enchaîne lyriquement: Je vous défends de lire sans verser des larmes, le récit des angoisses de la famille royale durant le voyage de Varennes..., le martyre de Louis XVII sous les coups de l'affreux Simon. ${ }^{39}$

On recommande ailleurs des auteurs moins récents comme Mézeray, le père Daniel, Sismondi ou des historiens qui semblent n'avoir eu comme qualité que celle d'être catholiques comme l'abbé Genoude ou Laurentie. Quelques commentaires dans le même article sur Michelet, alors au comble de la gloire en France, valent d'être cités ici :

38 Ibid., 15 et 24 février, 2 mars 1860. L'Ami de la Religion semble la principale source des nouvelles européennes de l'Abeille à cette époque.

39 Ibid., 5 décembre 1859. 
Aujourd'hui même, il est presque le veau d'or de la jeunesse, et je dirais, le mignon de la presse prête à bouleverser pour lui terre et mer... Les deux premiers volumes de son histoire de France firent du bruit grâce aux systèmes du philosophe allemand Hégel (sic) qui exaltèrent les jeunes imaginations... Enfin, cet écrivain a tour à tour, de la finesse, de la pénétration, de la bizarrerie, de la gêne, de la puérilité même dans ses comparaisons; beaucoup d'intérêt et de vérité, de la pénétration, des contradictions manifestes. ${ }^{40}$

Ainsi, on trouve à côté d'une sympathie très grande pour les historiens catholiques, une méfiance farouche à l'égard de ceux qui apparaissent antireligieux. Méfiance aisément explicable à une époque de luttes très vives dans lesquelles la rigoureuse méthode historique ne trouvait pas toujours son compte.

L'histoire, dira plus tard Mgr Dupanloup, est un de ces terrains que la presse anti-religieuse contemporaine a choisis de préférence et qu'elle a le plus exploités; elle a fait mentir contre nous l'histoire elle-même. ${ }^{41}$

Et ailleurs le même auteur continue:

Dans cet immense amas de productions historiques nées depuis trente ou quarante ans... on compte facilement les livres qui peuvent être mis entre les mains de la jeunesse, même aux mains de quiconque n'est pas assez fortement enraciné et fondé dans la foi, pour n'avoir rien à craindre des illusions de la fausse science. ${ }^{42}$

L'enseignement de l'histoire nationale entre dans une phase brillante après la publication des œuvres de Garneau. Un éditorial de l'Abeille en 1854 souligne le fait que «nous voyons aujourd'hui se réveiller le goût pour notre histoire.» Et l'auteur s'exclame plus loin avec justesse:

Mais quoi! nous saurions l'histoire du Mède et du Perse, du Grec et du Romain; nous saurions l'his-

40 Ibid., 22 décembre 1848.

$41 \mathrm{Mgr}$ l'évêque d'Orléans, op. cit., III : 227.

42 Ibid., II : 18. 
toire des temps et lieux séparés de nous par de nombreux siècles et par des milliers de lieues, et nous ignorions les hauts faits de notre patrie? nous foulerions une terre si fertile en grands souvenirs sans être capables de les recueillir. ${ }^{43}$

Dans un autre article, le même auteur, après une brève révision de l'histoire du Canada, termine par un appel à des 《correspondances sur notre histoire nationale ». ${ }^{44}$ Le journal publiera de nombreux articles se rapportant à notre histoire. ${ }^{45}$ L'abbé Laverdière au printemps de 1859 édite dans 26 livraisons successives une série de documents originaux sur l'histoire du Canada. En 1853-54, l'Abeille publie le «Récit des Voyages et découvertes du P. Jacques Marquette, de la compagnie de Jésus en l'année 1673, et aux suivantes ». On reproduit un extrait de Garneau en 1852 qui raconte la bataille de Montmorency. ${ }^{46}$ Même les fervents de l'histoire locale trouvent certains articles propres à les intéresser telles les notes historiques sur la Baie Saint-Paul publiées dans six livraisons en 1859-60.

Nous connaissons toutefois peu de choses au sujet du contenu des cours. Des notes d'élèves prises en 1853 nous révèlent que le professeur utilise alors Smith, Bibaud et Garneau. ${ }^{47}$ L'histoire qui s'étend jusqu'aux événements très contemporains se termine par des considérations sur les constitutions britannique et canadienne. ${ }^{48}$ Il est d'autant plus difficile de connaître ce qu'on enseigne qu'il semble que l'usage de manuels en histoire du Canada n'ait commencé au Petit Séminaire qu'en 1869. L'historien Garneau avait bien publié un abrégé de son Histoire qui connut un succès considérable alors. ${ }^{49}$ Mais il ne semble pas

43 L'A beille, 23 février 1854.

44 Ibid., 2 mars 1854.

45 Une compilation des articles de l'Abeille intéressant l'histoire du Canada a été publiée dans BRH, XXXIV (1928) : 607-615.

${ }^{46} L^{\prime}$ Abeille, 17 juin et 5 juillet 1852.

47 ASQ. M-94.

48 Ibid., 51-57.

49 Il en aurait été « distribué plus de vingt mille exemplaires 》, écrit Chauveau (François-Xavier Garneau, Sa vie et ses ouvres, CXXXII) qui ajoute: «Ce petit livre a contribué à développer le sentiment national autant et peut-être plus que le grand ouvrage. 


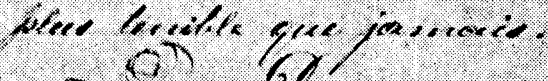



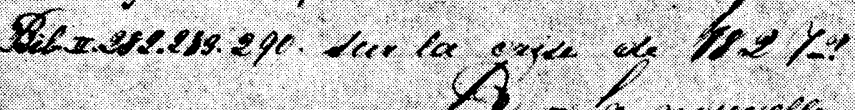

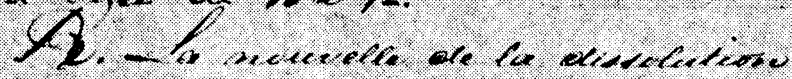



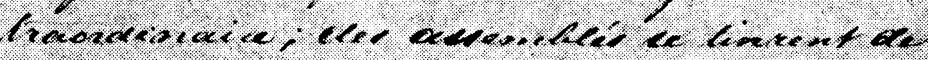

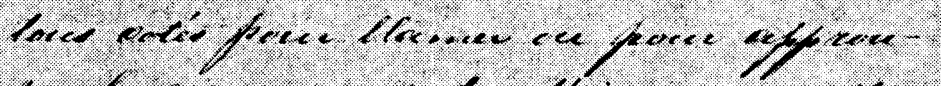

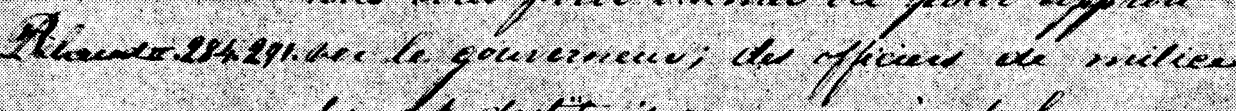

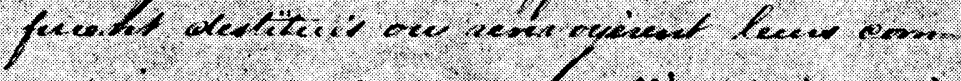

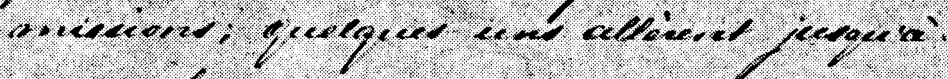

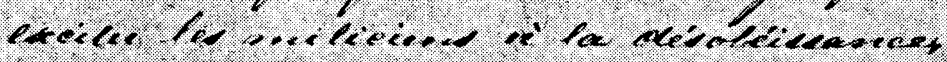

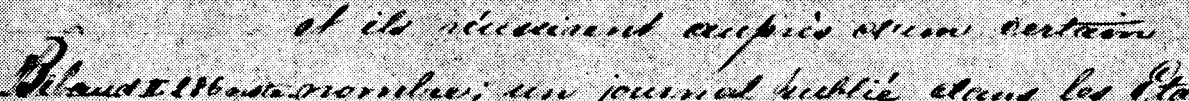
3)

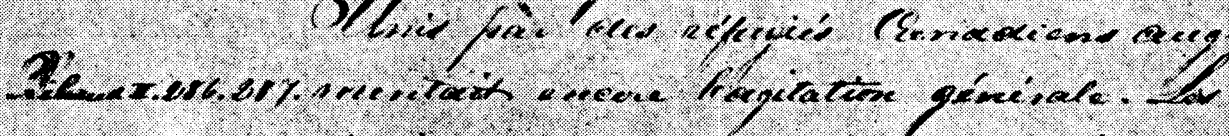

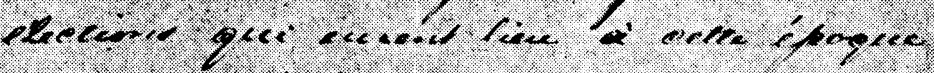

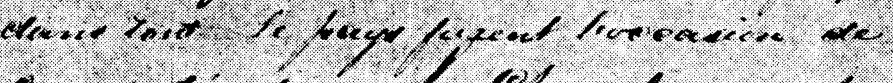

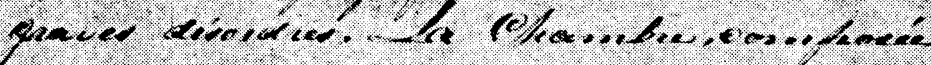

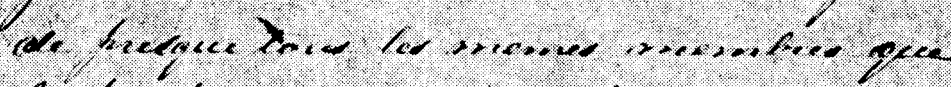

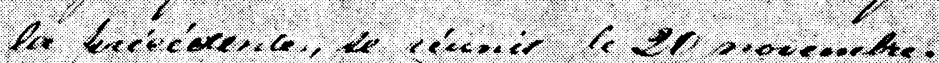

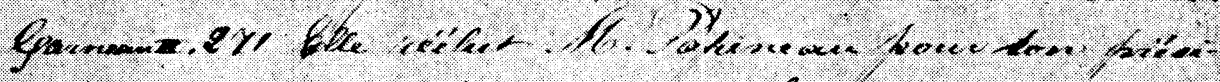

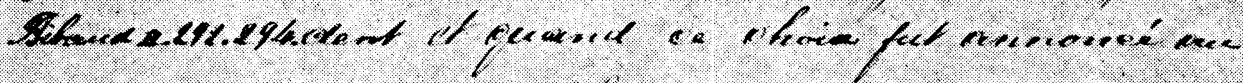

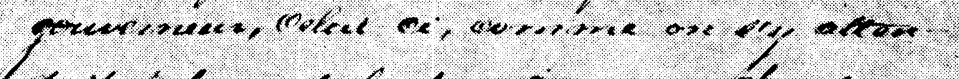

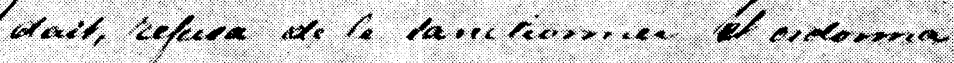

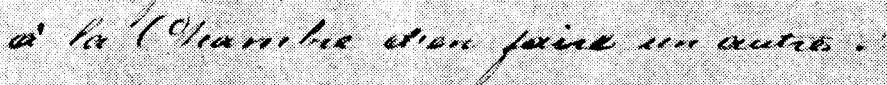

Une page d'un cahier de notes d'Histoire du Canada d'après un cours donné par l'abbé Taschereau en 1854 (ASQ. M-94). Dans la marge, renvois à Bibaud et à Garneau (2e éd.). 
qu'on l'ait utilisé au Séminaire, les professeurs préférant dicter leurs cours qu'ils préparaient personnellement.

En 1869, l'abbé Laverdière publia son Histoire du Canada. Ce travail honnête, bien au point quant aux connaissances historiques de l'époque, a été sévèrement jugé au point de vue pédagogique et littéraire. "Compilation assez indigeste de faits presque exclusivement militaires » qui « escamotait ou à peu près l'histoire de la domination anglaise », lui reprochera-t-on plus tard. ${ }^{50}$ Et Mgr Camille Roy concède tout au plus «qu'il pourrait servir de base à celui qui voudrait bien un jour doter enfin notre enseignement secondaire d'un bon manuel ».51 Ce petit livre fut utilisé dans les collèges classiques jusqu'au début du vingtième siècle. Le manuel de l'abbé Laverdière ne contenait aucune carte. La première carte pour servir à l'enseignement de l'histoire du Canada ne paraîtra d'ailleurs qu'en $1875 .{ }^{52}$ Le Séminaire en achète 6 exemplaires la même année.

Disons pour terminer que cette époque qui voit l'apparition de professeurs spéciaux d'histoire est également celle où se fixent les programmes à peu près dans la forme que nous leur connaissons aujourd'hui. Le tableau suivant qui compare le programme 1952 et celui de 1856 fera ressortir les similitudes. ${ }^{53}$

Sixième

(Eléments latins)

Cinquième

(Syntaxe)

Quatrième

(Méthode)
1856

1856
Histoire ancienne
(première partie)
Histoire ancienne
(deuxième partie)
Histoire romaine

1952

Orient ancien et Grèce

Histoire romaine

Histoire du Canada

Histoire du Moyen Age

50 L'abbé Chartier qui, écolier, étudia dans ce manuel. Cité dans un article du chanoine Groulx dans RHAF, VII (1952-53) : 435. naire, 72 .

51 Abbé Camille Roy, L'Université Laval et les fêtes du cinquante-

52 La première carte scolaire de la Nouvelle-France dans BRH, LII (1946) : 336. En 1865-66 l'enseignement de l'histoire nationale au Petit Séminaire se faisait encore sans carte au témoignage d'un ancien élève du temps. Cf. David Gosselin, Les étapes d'une classe au Petit Séminaire de Québec, 1859-1868, 159.

53 Annuaire de l'Université Laval pour l'année académique 1856-57. Programme des Etudes [du Séminaire] 55-56. Université Laval, Cours classique des garçons. Règlement de l'immatriculation et du baccalauréat. Programme des études, No 20 [1952], 41-52, 51-82. 
1856

Troisième

(Versification)

Seconde

(Belles-Lettres)

Première

(Rhétorique)
Histoire du Moyen Age

Histoire moderne

Histoire du Canada
1952

Temps modernes

Histoire contemporaine

Histoire du Canada (régime français)

Histoire du Canada (depuis 1763)

Histoire contemporaine

L'Histoire Sainte, enseignée en septième, sera plus tard annexée par l'histoire ancienne. Ce n'est qu'en 1891 qu'apparaîtra l'histoire «contemporaine ». Les programmes à cette date renferment par exemple les questions sur l'invasion des Etats Pontificaux, sur la reine Victoria et sur Guillaume II, empereur d'Allemagne.

PierRe SAVARD, assistant à la Faculté des Arts (Québec)

\section{B I B L I O G R A P H I E}

\section{I - SOURCES}

A - Manuscrites

1 - Cahiers de notes d'élèves

2 - Cartes et divers autres documents.

$\mathrm{B}$ - Imprimées

1 - Manuels

2 - Prospectus, catalogues et annuaires

3 - Journaux

4 - Autres sources imprimées.

II - GUIDES ET INVENTAIRES

III - ETUDES

IV - PÉRIODIQUES

\section{I - SOURCES}

\section{A - SOURCES MANUSCRITES}

\section{1 - Cahiers de notes d'élèves (ASQ)}

Nous avons dépouillé aux Archives du Séminaire de Québec une trentaine de cahiers d'élèves portant sur l'histoire et la géo- 
graphie. La plupart reproduisent très fidèlement les cours donnés et constituent ainsi une source inestimable. Toutefois plusieurs sont incomplets ou parfois se répètent. De plus on ne dispose souvent d'aucun cahier pour une période assez longue. Enfin certains de ces cahiers sont sûrement venus d'autres collèges et ainsi ne nous sont à peu près d'aucune utilité tandis qu'un bon nombre concernent l'histoire ecclésiastique enseignée au Grand Séminaire.

On trouvera ici la liste de ceux que nous avons cités au cours de notre travail:

M-272. 1790. Morceaux choisis. Géographie en vers. Par: le Père Buffier, s.j. 23p.

M-978. 1801. Principes de géographie. Professeur: abbé Pierre Bossu. 14p.

M-978. 1800, copié en 1804. Calendrier romain. 4p. Egypte 22p. Professeur: abbé Joseph Signay.

M-95. Cours abrégé de Belles-Lettres.

M-61. 1821. Cours abrégé d'histoire ancienne. Professeur: abbé Jean-Baptiste Lahaille. Notes de Léon Gingras.

M-55. 1828. Cours d'histoire d'Angleterre. Notes de L. Prévost. $113 \mathrm{p}$.

M-56. 1828. Cours d'histoire d'Angleterre. Notes d'EdouardGabriel Plante. 208p. Reproduit le précédent à quelques lignes près.

M-53. 1843. Cours abrégé d'histoire du Canada. Notes de François-Gaspard Drolet. Le professeur serait l'abbé Holmes. 123p.

M-94. 1854. Cours abrégé d'histoire du Canada sous la domination anglaise. Professeur: abbé E.-A. Taschereau. 57p. Le premier cours couvrant la période qui suit 1763.

Nous avons également consulté:

Polygraphie 19, 48: «Sommaire qui remonte à la domination française. Embryon d'un cours d'histoire du Canada 14991760. 》

\section{2 - Cartes et divers autres documents (ASQ)}

Les Archives du Séminaire conservent deux précieuses séries de cartes: 
Tiroir 223 des cartes:

Atlas I. [-Atlas] "pour servir à la Géographie du Petit Séminaire de Québec, 1804». Série de 41 cartes détachées, manuscrites et coloriées.

Atlas 2. 5 cartes du XVIIIe s. de Delisle etc. 7 cartes pour l'enseignement de l'histoire sainte. Ces cartes toutes datées d'avant 1792 ont probablement servi à l'enseignement.

C'est également aux archives du Séminaire qu'on conserve le catalogue de la Bibliothèque de 1782:

A. G. Dudevant, ptre. Catalogue des livres de la bibliothèque du Séminaire des Missions Etrangères de Québec fait dans le mois de May 1782.

Nous avons également utilisé un grand cahier, compilation de l'abbé Amédée Gosselin et de ses successeurs:

Supérieurs, Directeurs, Officiers et professeurs du Séminaire de Québec de 1663 à 1860. Malgré son titre, cette précieuse compilation renferme des renseignements jusqu'à 1940.

Enfin deux autres compilations de l'abbé Gosselin nous ont rendu de précieux services pour l'établissement des programmes de jadis. Il s'agit de deux cahiers de copie manuscrite dont l'ensemble totalise environ 350 pages :

Séminaire de Québec. Thèse de Mathématiques. Exercices littéraires. Programmes des Etudes. Comptes rendus des journaux. Distribution des Prix. De 1770 à 1840. Extraits des journaux et particulièrement de la Gazette de Québec, du Canadien, du Vrai Canadien, du Courrier de Québec. Fait en Déc. 1908. A.E.G.

Séminaire de Québec. Exercices littéraires, programmes d'études, comptes rendus du Canadien, Distribution des prix, etc. De 1841 à 18:45. Suite du précédent.

\section{B - SOURCES IMPRIMEES}

\section{1 - Manuels}

La Bibliothèque de l'Université Laval conserve encore un nombre considérable de manuels d'histoire et de géographie utilisés au Petit Séminaire de Québec autrefois. On trouvera énumérés ici seulement ceux que nous citons au cours de notre travail:

[Abbé François Pigeon]. Géographie à l'usage des écoliers du Petit Séminaire de Québec. Québec, Neilson, 1804. [4]$27-[1]$ p. $10 \mathrm{~cm}$. 
[Anonyme]. Géographie à l'usage du Séminaire de Nicolet. Montréal, Imprimé par James Lane, 1828. 49p. $18 \mathrm{~cm}$.

[Abbé Jean Holmes]. Nouvel abrégé de géographie moderne suivi d'un petit abrégé de Géographie ancienne à l'usage de la Jeunesse. Québec, Neilson et Cowan, 1831. XII-51p. $18 \mathrm{~cm}$.

- Nouvel abrégé de géographie moderne, suivi d'un abrégé de géographie sacrée à l'usage de la jeunesse; en deux parties. Québec, Neilson et Cowan, 1832. XII-159p. $18 \mathrm{~cm}$.

- Nouvel abrégé de géographie moderne, suivi d'un appendice et d'un abrégé de géographie sacrée à l'usage de la jeunesse. Seconde édition. Québec, Neilson et Cowan, 1833.

Paginé de 161 à 277 avec en plus XXX-16 pages. Il s'agit de la suite de la publication précédente.

M. Moustalon. Abrégé de Mythologie à l'usage des maisons d'éducation. Québec, Neilson et Cowan, 1832 . 55p. $11 \mathrm{~cm}$.

[Anonyme]. Histoire romaine, depuis la fondation de Rome jusqu'à la fin de l'empire d'Occident; à l'usage des maisons d'éducation [...] Lyon, Rusand, Paris, Librairie ecclésiastique, 1832. 232p. $14 \mathrm{~cm}$. Cartes. Il s'agit d'une réédition.

[Anonyme]. Histoire romaine, depuis la fondation de Rome jusqu'à la fin de l'empire d'Occident; à l'usage de la jeunesse. Nouvelle édition revue et corrigée. Québec, Cary, 1832.272p. $14.5 \mathrm{~cm}$. Réimpression de l'ouvrage précédent.

[Anonyme]. Abrégé chronologique d'histoire sacrée et profane à l'usage du Collège de Montréal. Montréal, Leclerc et Jones, imprimeurs, 1835. [2] 101-[III]p. $17.5 \mathrm{~cm}$.

[Anonyme]. Nouvel abrégé de l'histoire de France depuis Pharamond jusqu'à nos jours; à l'usage de la jeunesse. Québec, Cary, 1837. $82 \mathrm{p} .17 .5 \mathrm{~cm}$.

\section{2 - Prospectus, catalogues et annuaires}

Entre 1831 et 1842 le Séminaire publie à peu près chaque année un prospectus intitulé Exercices philosophiques et littéraires [...] qui donne le programme de la séance publique de fin d'année ainsi que le programme des études. Certaines années, cette publication est intitulée Programme abrégé du cours d'étude du Petit Séminaire de Québec pour l'année [...] Ces prospectus sont souvent reproduits tels quels dans les journaux du temps avec l'annonce ou le compte rendu des séances.

Pour la période qui va de 1847-48 à 1855-56 nous disposons de neuf précieuses publications intitulées: Catalogue des officiers 
et des élèves du Petit Séminaire de Québec [...] On y trouve la liste des élèves par classe, celle des professeurs, des renseignements sur le programme d'étude, les associations, etc. De 24 à 49 pages chacun. Publiés annuellement et imprimés sur les presses de l'Abeille.

En 1856-57 paraît le premier Annuaire de l'Université Laval. On y trouve de temps à autre des renseignements sur les questions d'examens et les programmes d'étude au Petit Séminaire. Publication annuelle. En 1869, première édition des Programmes $d u$ Baccalauréat ès Arts à l'Université Laval (Québec, Delisle, 1869. 41 p. $18.5 \mathrm{~cm})$. Plusieurs rééditions correspondant à chaque remaniement du programme jusqu'à la dernière:

Université Laval. Cours classique des garçons. Règlement de l'immatriculation et du baccalauréat. Programme des Etudes. No 20. Québec, 1952. 128p. 22cm.

\section{3 - Journaux}

Parmi les journaux du temps qui donnent des comptes rendus des séances publiques nous avons consulté:

La Gazette de Québec, 17 août 1809, 23 août 1810.

Le Canadien, 3 et 5 juillet 1833, 16 août 1833, 3 octobre 1834 (critique de Ferland sur l'ouvrage de Lebrun), 14 août 1835, 14 août 1839,16 août 1841 .

Mais nous avons surtout utilisé l'Abeille, journal hebdomadaire, fondé en octobre 1848 p?r les écoliers et qui paraît juscu'à 1881 avec des interruptions plus ou moins longues (1848-1854; $1858-1862 ; 1877-1881)$.

\section{4 - Autres sources imprimées}

AUBERT DE GASPE, Philippe. Les Anciens Canadiens. 2 vol., Québec, Côté, 1877 . 298 et $240 \mathrm{p} .17 \mathrm{~cm}$.

BRUNET, Michel. FREGAULT, Guv, TRUDEL, Marcel. Histoire du Canada par les textes. Paris-Montréal, Fides, 1952. 298p. 24cm. Voir p. 67-81, le mémoire attribué à Hocquart intitulé Le Canada pendant la paix de Trente Ans.

GOSSELIN, l'abbé David. Les étapes d'une classe au Petit Séminaire de Québec 1859-1868. Québec, H. Chasse, 1908. $291-I I p .20 \mathrm{~cm}$.

HOLMES, abbé Jean. Conférences de Notre-Dame de Québec. Québec, Darveau, 1875 . 212p. $19 \mathrm{~cm}$. 
HOUSTON, James. Le Répertoire national ou recueil de littérature canadienne. Montréal, Lovell et Gibson, 18481850. I, VIII-376p., II, 384p., III, 387p., IV, 411p. $21 \mathrm{~cm}$.

LEBRUN, Isidore. Tableau statistique et politique des Deux Canadas. Paris, Treuttel et Wurtz, 1833. [4] 588 [2]p. $21 \mathrm{~cm}$.

Mandements, lettres pastorales et circulaires des évêques de Québec publiés par Mgr H. Têtu et l'abbé C.-O. Gagnon. Québec, Imprimerie générale A. Côté et Cie, 1888. Vol. II: 1741-1806. 566p. $23 \mathrm{~cm}$.

Revue de l'Université Laval. Documents pour servir à l'histoire du Séminaire de Québec. Nous avons utilisé le document no CXV : Plan d'éducation du Séminaire de Québec, octobre 1790 (RUL, XIII (1958-59) : 270-274) et le document no CXXVI: Plan d'éducation du Séminaire de Québec 1816 (RUL, XIII (1958-59) : 302s).

ROLLIN, Charles. Traité des Etudes ou de la manière d'enseigner et d'étudier les Belles-Lettres. Paris, Chamerot, 1845. VIII-879p. $27.5 \mathrm{~cm}$. Index.

ROY, J.-Edmond. Souvenirs d'une Classe au Séminaire de Québec (1867-187\%). Lévis, Imprimerie de l'auteur, 1905. 529 p. $22 \mathrm{~cm}$. L'auteur utilise largement l'Abeille. Il annonce un second volume qui n'a jamais vu le jour.

TOCQUEVILLE, Alexis-Charles-Henri-Maurice Clérol de. Oeuvres, papiers et correspondances. Edition définitive publiée sous la direction de J.-P. Mayer. Paris, Gallimard, 1951. Vol. I (2 tomes) De la démocratie en Amérique. XLIX-466 et $399 \mathrm{p} .23 \mathrm{~cm}$.

\section{II - GUIDES ET INVENTAIRES}

FARIBAULT, G.-B. Catalogue d'ouvrages sur l'histoire de l'Amérique et en particulier sur celle du Canada [...] Québec, Cowan, 1837. [4]-207p. $22 \mathrm{~cm}$.

GAGNON, Philéas. Essai de bibliographie canadienne. Inventaire d'une bibliothèque [...] Québec, Imprimé par l'auteur. 2 volumes: I:X-711p., publié en 1895; II: XI-[3]462p., publié en $1913.25 \mathrm{~cm}$.

HAMELIN, Louis-Edmond. Bibliographie annotée concernant la pénétration de la géographie dans le Québec: I: les Manuels. Dans les Cahiers de Géographie de Québec, $4^{\mathrm{e}}$ année, no 8 (avril-septembre 1960) : 345-358. 


\section{III - ÉTUDES}

[Anonyme]. Le Séminaire de Nicolet. Montréal, La Minerve, $1867.214 \mathrm{p} .21 .5 \mathrm{~cm}$.

[Anonyme]. Les Ursulines de Québec depuis leur établissement jusqu'à nos jours. 4 vol. Québec, Darveau, 1864-1866.

AUDET, Louis-Philippe. L'éducation au temps de Monseigneur de Laval dans La Société Canadienne d'Histoire de l'Eglise Catholique, rapport 1957-58: 59-78.

BIBAUD Jeune [Maximilien]. Tableau historique des progrès matériels et intellectuels du Canada. Montréal, Cabinet de lecture, $1878.50 \mathrm{p} .18 .5 \mathrm{~cm}$.

BLANCHARD, Raoul. L'Est du Canada français « Province de Québec ». 2 vol. Montréal, Beauchemin, Paris, Masson, 1935. 26cm. Ill. Cartes.

Centenaire de l'Histoire du Canada de François-Xavier Garneau. Deuxième semaine d'histoire à l'Université de Montréal, 23-27 avril 1945. Montréal, Société historique de Montréal, $1945.461 \mathrm{p} .23 \mathrm{~cm}$.

CHARTIER, Mgr Emile. Au Canada francais. La vie de l'esprit, 1760-1925. Montréal, Valiquette, $1941.354 \mathrm{p} .19 \mathrm{~cm}$. CHAUVEAU, P.-J.-O. François-Xavier Garneau. Sa vie et ses œuvres. Montréal, Beauchemin \& Valois, 1883. 282p. $22 \mathrm{~cm}$.

CHOQUETTE, chanoine C.-P. Histoire du Séminaire de Saint-Hyacinthe depuis sa fondation jusqu'à nos jours. 2 vol. Montréal, Imprimerie de l'Institution des Sourds-Muets, 1911-12. 538 et $403 \mathrm{p} .23 \mathrm{~cm}$. Ill. Index.

DARVEAU, L.-M. Nos hommes de lettres. Montréal, Stevenson, 1873. [6]-VI-280p. $19 \mathrm{~cm}$.

DIONNE, N.-E. Vie de C.-F. Painchaud, prêtre, curé, fondateur du collège de Sainte-Anne-de-la-Pocatière. Québec, Brousseau, $1894.440 \mathrm{p} .22 \mathrm{~cm}$.

[DUPANLOUP, Mgr] Mgr l'évêque d'Orléans. De la haute éducation intellectuelle. 3 vol. Paris, Douniol, 1864-66. 600, 604 et $653 \mathrm{p} .22 \mathrm{~cm}$.

FAUCHER DE SAINT-MAURICE, Henri-Edmond. L'abbé C.-H. Laverdière. s.l., n.d. 9p. Tiré de l'Opinion publique du 27 mars 1873.

FAUTEUX, Aegidius. Les Bibliothèques Canadiennes. Etude historique. Extrait de la «Revue Canadienne». Montréal, Arbour et Dupont, 1916. $45 \mathrm{p} .25 \mathrm{~cm}$. 
FERLAND, abbé Jean-Baptiste. Mgr Joseph-Octave Plessis, évêque de Québec. Québec, Brousseau, 1876. 288p. $16.5 \mathrm{~cm}$.

FORGET, abbé Anastase. 1833 - Un siècle - 1933. Histoire du Collège de l'Assomption. Montréal, Imprimerie populaire, 1933. $813 \mathrm{p} .26 \mathrm{~cm}$. Ill.

FUETER, Edvard. Histoire de l'historiographie moderne [...] traduit de l'allemand par Emile Jeanmaire (avec notes et additions de l'auteur). Paris, Alcan, 1914. $785 \mathrm{p} .25 \mathrm{~cm}$. GALARNEAU, Claude. L'enseignement des humanités du XVIe au XVIIIe siècle. Mémoire présenté à la Commission du Programme de la Faculté des Arts de Laval [...] 1958. 20p. Ronéotypé.

GAUDIN, A. C. The educational views of Charles Rollin. New-York, 1939.

GOSSELIN, Abbé Amédée. L'Instruction au Canada sous le Régime francais (1635-1760). Québec, Laflamme et Proulx, 1911. 501 p. $23 \mathrm{~cm}$.

GOSSELIN, Abbé Auguste-Honoré. Un bon patriote d'autrefois. Le Docteur Labrie. Québec, Laflamme et Proulx, 1907 (nouvelle édition). XVI-274p. $19 \mathrm{~cm}$.

GREARD, Oct[ave]. Education et instruction. Enseignement secondaire. 2 vol. Paris, Hachette, 1889. (2e éd.) 351 et $359 \mathrm{p} .18 \mathrm{~cm}$.

GROULX, abbé Lionel. L'enseignement francais au Canada. 2 vol. Vol. I: Dans le Québec. Montréal, Librairie d'action canadienne-française limitée, $1931.327 \mathrm{p} .24 \mathrm{~cm}$.

- Notre maître le passé (2e série). Montréal, Granger, 1936. $309 \mathrm{p} .19 \mathrm{~cm}$.

HAZARD, Paul. La pensée européenne au XVIIIe siècle de Montesquieu à Lessing. 3 vol. Paris, Boivin, $1946.23 \mathrm{~cm}$. Ill. JULLIAN, Camille. Extraits des historiens français au $X I X e$ siècle publiés, annotés et précédés d'une introduction sur l'histoire de France. Paris, Hachette, 1904 (3e éd. revue). CXXVIII-684p. $15 \mathrm{~cm}$. L'introduction fournit une synthèse encore valable sur l'historiographie française au siècle dernier.

LARUE, Hubert. Mélanges historiques et littéraires et d'économie politique. Vol. II. Québec, Delisle, $1881.275 \mathrm{p} .23 \mathrm{~cm}$. Eloge historique de Laverdière (extrait) : 145-151.

MAHEUX, abbé Arthur. Notes historiques sur le cours classique. Mémoire présenté à la Commission du Programme de la Faculté des Arts de Laval [...] 1958. 22p. Ronéotypé. 
MARROU, Henri-Irénée. De la Connaissance historique. Paris-Louvain, Publications universitaires de Louvain et Editions du Seuil, 1954.300 p. $20 \mathrm{~cm}$.

MAURAULT, Olivier. Le Petit Séminaire de Montréal. Montréal, Derome, 1918. 240p. $23.5 \mathrm{~cm}$.

MILLER, Emile. Pour qu'on aime la géographie. Montréal, Ducharme, $1921.247 \mathrm{p} .19 .5 \mathrm{~cm}$.

MONROE, Walter S. editor. Encyclopedia of Educational Research. New York, The MacMillan Co., 1941. 1344p. 27.5 $\mathrm{cm}$.

MORGAN, Henry J. Sketches of Celebrated Canadians, and persons connected with Canada, from the earliest period in the history of the province down to the present time. Quebec, Hunter, Rose \& Co., London, Trubner \& Company [...] 1862. XIII-780-4p. $23 \mathrm{~cm}$.

O'BREADY, Mgr Maurice. John ou Jean Holmes, 1954. Travail dactylographié déposé à l'Université de Sherbrooke. $\mathrm{X}-213 \mathrm{p}$. Biographie romancée. Sans références. Bibliographie.

REINHARD, Marcel. Histoire de France. Ouvrage publié sous la direction de Marcel Reinhard avec la collaboration de Norbert Dufourcq. 2 vol. Paris, Larousse, 1954. Voir I: RCQ 24, Histoire des Histoires de France par Marcel Reinhard. Excellente revue de l'enseignement de l'histoire nationale en France à travers les âges.

ROCHEMONTEIX, P. Camille de. Un collège de Jésuites aux XVIIe \& XVIIIe siècles. Le Collège Henri IV de La Flèche. 4 vol. Le Mans, Leguicheux, 1889. 312, 333, 356 et $444 \mathrm{p} .21 .5 \mathrm{~cm}$. Ouvrage qui relève autant du genre polémique que de l'histoire.

ROY, Antoine. Les Lettres, les Sciences et les Arts au Canada sous le régime francais. Essai de contribution à l'histoire de la civilisation canadienne. Paris, Jouve, 1930. XVI-292p. $24 \mathrm{~cm}$.

ROY, abbé Camille. L'Université Laval et les fêtes du cinquantenaire. Québec, Dussault, 1903. VIII-395p. 24cm. Ill. Semaine d'Histoire du Canada. Première session tenue à la bibliothèque Saint-Sulpice à Montréal du 23 au 27 novembre 1925 sous les auspices de la Société historique de Montréal. Compte rendu et Mémoires. Montréal, Société historique de Montréal, 1926 . $456 \mathrm{p} .25 \mathrm{~cm}$. Consulter C.-J. Magnan, L'histoire du Canada au cours primaire 1825-1925, 344-376. 
SICARD, abbé Augustin. Les études classiques avant la Révolution. Paris, Perrin et Cie, 1887 . X-589p. $19 \mathrm{~cm}$.

TRUDEL, Marcel. L'influence de Voltaire au Canada. 2 vol. Montréal, Fides, 1945. 222 et 316p. 20.5cm. Bibliographie. Index.

- L'Eglise canadienne sous le Régime militaire 175.9-1764. Vol. I: Les problèmes. Montréal, Les Etudes de l'Institut d'histoire de l'Amérique française, 1956. XXXIII-362p. $22.5 \mathrm{~cm}$. Vol. II : Les Institutions. Québec, Les Presses Universitaires Laval, 1957. VII-492p. 22.5cm. Index. Cartes. VIATTE, Auguste. Histoire littéraire de l'Amérique française des origines à 1950. Québec, Presses Universitaires Laval, Paris, Presses Universitaires de France, 1954. XI$547 \mathrm{p} .19 \mathrm{~cm}$.

WEILL, Georges, Histoire de l'enseignement secondaire en France 1802-1920. Paris, Payot, 1920. 255p. $18.5 \mathrm{~cm}$. Bibliographie.

\section{IV - PERIODIQUES}

[Anonyme]. Le tableau statistique et politique de Isidore Lebrun dans BRH XLIV (1938): 183s.

CARON, abbé Ivanhoë. Le collège classique de Saint-Roch de Québec dans BRH XLV (1939) : 97-100.

DROLET, Antonio. Les éditions de l'abrégé de géographie de l'abbé Holmes dans BRH LII (1947): 160s.

- La bibliothèque du Séminaire de Québec et son catalogue de 1782 dans Le Canada français XXVIII (1940) : 261-266. GERIN-LAJOIE, Antoine. Biographie de l'abbé J.-B.-A. Ferland dans le Foyer Canadien III (1865) : I-LXXII. Québec, Bureaux du « Foyer Canadien 》, 1865.

GOSSELIN, abbé Auguste. Le vrai monument de Champlain: ses ouvres éditées par Laverdière dans MSRC (troisième série) II (1908) : 3-23.

GROULX, chanoine Lionel. Henri Bourassa et la chaire d'histoire du Canada à l'Université de Montréal dans RHAF VI (1952-53): 430-439. Sur le renouveau de l'histoire du Canada et de son enseignement dans les collèges classiques après 1900 .

LEBEL, Maurice. The teaching of History in Quebec Schools dans Culture XIX (1958) : 376-390. Bref résumé des débuts de l'enseignement de l'histoire nationale au cours secondaire: $377 \mathrm{~s}$. 
MAHEUX, abbé Arthur. Des souvenirs ... une expérience. Documents d'histoire du Canada dans l'enseignement secondaire dans ESC XXXVI (1956-57) : 277ss.

- Jacques Labrie (1784-1831) dans MSRC (troisième série) XLII (1948) : 119-131.

O'BREADY, Mgr Maurice. Un pédagogue dynamique dans Le Devoir du 14 mars 1957. Extrait du travail cité plus haut. ROY, Antoine. Les sources imprimées de l'histoire du Canada français [dans l'Abeille de 1848 à 1881] dans BRH XXIV (1928) : 607-615.

R [oy], P.-G. La première carte scolaire de la Nouvelle-Fran$c e$ dans BRH LII (1946) : 336.

SULTE, Benjamin. Nos ancêtres étaient-ils ignorants? dans MSRC (troisième série) XII (1918): 201-208.

TRUDEL, Marcel. A propos de cette unique grammaire de 1760 dans RUL IX (1954-55) : 780-785.

WADE, Mason. The contribution of abbé John Holmes to Education in the Province of Quebec dans Culture XV (1954) : 3-16.

PIERRE SAVARD 